\title{
FRATURAS PERIPROTÉTICAS EM ARTROPLASTIA TOTAL DE JOELHO
}

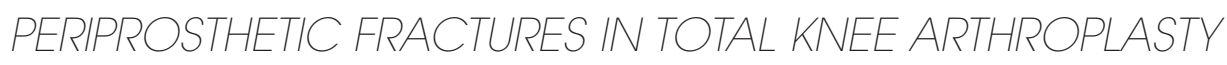

Paulo Gilberto Cimbalista de Alencar ${ }^{1}$, Giovani De Bortoli², Inácio Facó Ventura Vieira², Christiano Saliba Uliana ${ }^{2}$

\section{RESUMO}

A associação do maior número de artroplastias totais de joelho com a maior expectativa de vida da população tem levado a mais complicações de longo prazo, que se somam à baixa qualidade óssea dos pacientes mais idosos e culminam, muitas vezes, em fraturas periprotéticas. Este complexo problema ortopédico tem apresentação clínica muito variável, podendo acometer quaisquer dos ossos do joelho e levar a resultados desastrosos, em virtude de sua difícil solução. O seu tratamento exige do ortopedista amplo conhecimento tanto de técnicas de artroplastia como de osteossíntese, além de elaborado arsenal terapêutico como, por exemplo, acesso a banco de ossos.

Descritores - Fraturas periprotéticas; Artroplastia do joelho; Atualização

\section{ABSTRACT}

The increasing number of total knee arthroplasties, in combination with the population's longer life expectancy, has led to a greater number of long-term complications. These complications are also correlated with poor bone quality in the elderly and often result in periprosthetic fractures. This complex orthopedic problem has very diverse clinical presentation, possibly afflicting periprosthetic fracture may happen in any bone that constitutes the knee and, due to the difficulty of finding a solution, may lead to disastrous outcomes. The treatment demands broad knowledge from the orthopedic surgeon, not only regarding arthroplasty techniques, but also osteosynthesis, as well as an elaborate therapeutic including, for example, access to a bone bank.

Keywords - Periprosthetic fractures; Arthroplasty, replacement, knee; Atualization

As fraturas podem acometer os três ossos tratados pela ATJ, ou seja, patela, tíbia ou fêmur.

\section{Fraturas de patela}

As complicações pós-operatórias em ATJ mais frequentes envolvem o aparelho extensor de diversos modos, e fraturas de patela são um problema significativo. A incidência, segundo dados de literatura, varia de $0,11 \%$ a $21,4 \%{ }^{(1,2)}$. Diversas causas podem ser atribuídas: hiperpressão no aparelho extensor causada por componente femoral excessivamente anteriorizado ou por ressecção insuficiente da superfície articular patelar para colocação da prótese patelar (overstuffing); insuficiência vascular provocada por liberação do retináculo femoropatelar lateral, por efeito da cimentação, desnervação periférica da patela ou, ainda, por ressecção óssea excessiva da superfície articular; desalinhamento do aparelho extensor decorrente, na maioria das vezes,

1 - Chefe do Grupo de Cirurgia do Quadril e Joelho do Hospital de Clínicas da Universidade Federal do Paraná.

2 - Pós-Graduando do Grupo de Cirurgia do Quadril e Joelho do Hospital de Clínicas da Universidade Federal do Paraná.

Correspondência: Av. Nossa Senhora Aparecida, 283 - 80440-000 - Curitiba-PR, Brasil. E-mail: pa@clinicapauloalencar.com 
da rotação incorreta do componente femoral e/ou tibial; uso de prótese patelar com plug de fixação único, central e de diâmetro grande; decorrência de fatores específicos do paciente, como obesidade, atividade física excessiva, hábito de hiperflexionar o joelho; e, finalmente, em consequência a trauma após a operação ${ }^{(1,3-12)}$ (Figura 1).

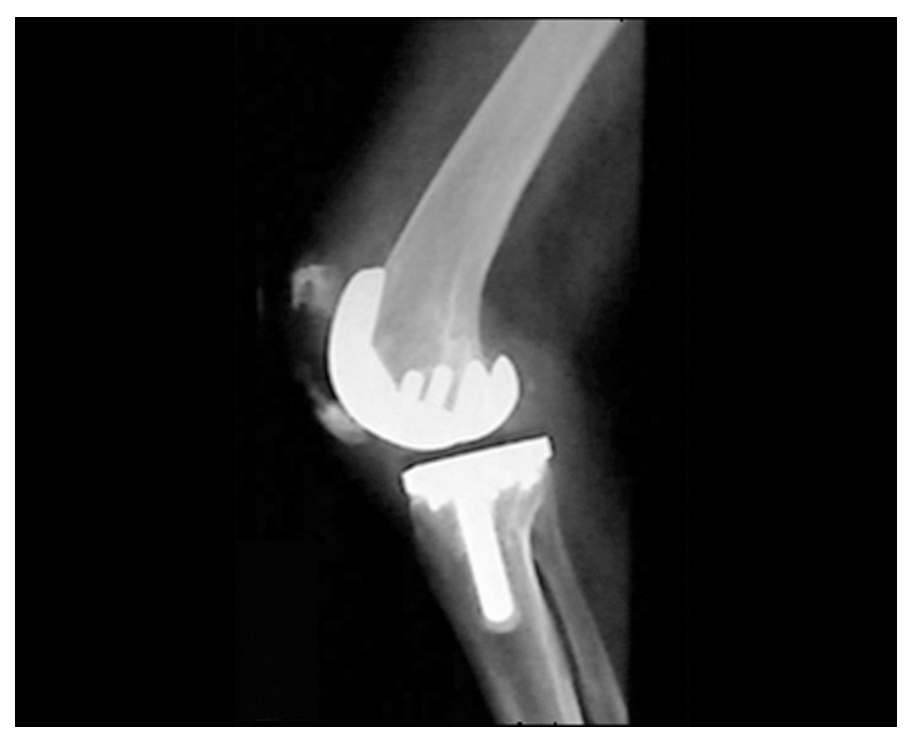

Figura 1 - Fratura de patela

A apresentação mais frequente é a de achado radiográfico nas visitas de controle pós-operatório, absolutamente assintomáticas. Nessas situações, se a função do aparelho extensor estiver preservada, habitualmente apenas se continua acompanhando o paciente de maneira periódica ${ }^{(1,6,13-15)}$.

De modo geral, o tratamento deve ser selecionado levando-se em conta os achados de exame físico. Caso o aparelho extensor esteja íntegro e com alinhamento adequado e componente patelar fixo ao osso no exame radiográfico, deve ser orientado tratamento conservador, com imobilização para alívio dos sintomas por seis semanas $^{(1,3,6,13,15)}$. Nas demais situações pode ser indicado tratamento cirúrgico: fixação da fratura de patela com sistema de banda de tensão ou uso de âncoras ósseas, no caso de pequenos fragmentos nos polos da patela. Esses procedimentos são tecnicamente difíceis no caso de fratura da patela em que há uma prótese, e podem ser acrescidos de problemas adicionais se houver osteólise ou soltura do componente patelar. Na literatura, os relatos sobre tratamento cirúrgico de FxP de patela são de altas incidências de complicações, levando no final, muitas vezes, a procedimentos de salvação, como artrodeses ou ressecção artroplástica do joelho.

Modificações na técnica cirúrgica, como especial atenção à rotação dos componentes e redução no nú- mero de liberações retinaculares, têm trazido redução na incidência de fraturas patelares. Também contribuíram algumas mudanças no design dos componentes, como o sulco patelar do componente femoral mais profundo e a modificação do sistema de fixação do componente patelar ao osso, com três pegs pequenos ao invés de um central de grande diâmetro ${ }^{(3,5,9,16,17)}$.

Cabe comentar que a experiência de vários serviços em não substituir a superfície articular da patela resultou em redução acentuada das fraturas de patela pós ATJ, como no caso do serviço dos autores. Em 10 anos de experiência e mais de 2.000 ATJ em que não foram utilizados componentes patelares, não houve fratura de patela até o momento da redação deste artigo.

\section{Fraturas da tíbia}

Fraturas periprotéticas da tíbia são raras; conforme relatado por Healy, de 1970 até 1992, somente 32 casos haviam sido descritos $^{(16)}$. Neste mesmo período, a maior série publicou 15 casos $^{(17)}$. Posteriormente, em 1997, Felix et $a l^{(18)}$ apresentaram 102 casos. Em grande número de vezes ocorre durante a operação de revisão da ATJ, e geralmente sem desvio. Nas demais situações pode estar associada a osteólise local ou soltura asséptica do componente tibial. Muitos pacientes que não são acompanhados regularmente podem apresentar perdas ósseas consideráveis, e o retorno ao médico pode ser em decorrência da FxP.

Na ocorrência da fratura intraoperatória devem ser feitas radiografias para verificar a natureza e extensão do problema. Em casos de perfurações inadvertidas ou havendo perda óssea pode estar indicado o uso de enxertos ósseos homólogos. As fraturas geralmente não são desviadas e a própria haste intramedular do componente tibial pode ser suficiente para manter a redução e estabilidade até a consolidação.

Quando ocorrem após vários anos da ATJ, as perdas ósseas decorrentes da mobilização do componente tibial solto ou da osteólise causadas pelas partículas resultantes do desgaste devem ser repostas. Os defeitos podem ser preenchidos por cimento metilmetacrilato, blocos e cunhas metálicos ou enxerto ósseo de banco de tecidos. De acordo com a natureza do defeito, se este for cavitário utiliza-se tecido ósseo moído para preenchimento, e se for segmentar (envolvendo parte significativa da cortical tibial) é empregado tecido ósseo estrutural, geralmente obtido da tíbia proximal de um doador cadáver. O uso de haste intramedular no componente tibial é mandatório nesses casos, com finalidade de fixação do implante em um segmento ósseo estável e para proteção do enxerto ósseo aos esforços mecânicos ${ }^{(19,20)}$ (Figuras 2a, b, c). 

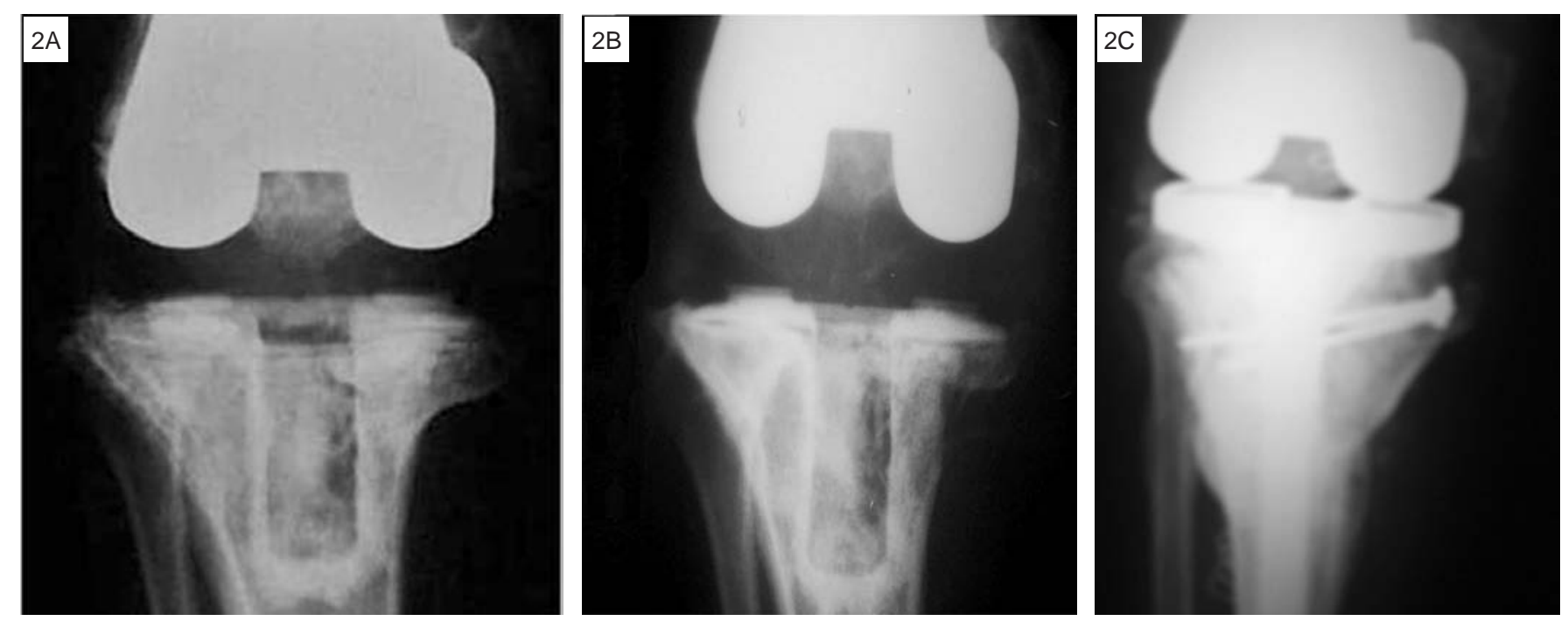

Figura 2 - A) Prótese total de joelho pós-operatório imediato, com boa qualidade óssea. B) Osteólise de tíbia proximal com fratura periprotética. C) Tratamento com revisão da ATJ e enxerto homólogo de tíbia proximal, fixado com parafusos

\section{Fraturas de fêmur}

As fraturas de fêmur distal após ATJ são as mais estudadas na literatura, devido a sua incidência $(0,3$ a 7,8\%) ${ }^{(21,22)}$ e importância clínica.

As causas de risco estão relacionadas na Tabela 1. É interessante observar que os defeitos ósseos na cortical femoral causados durante a confecção dos cortes para implantação do componente femoral (notch) têm sido questionadas como causa de FxP. Naturalmente, depende da extensão do defeito iatrogênico, mas em nossa experiência pessoal e de outros autores ${ }^{(23)}$ não houve nenhum caso de FxP nos casos em que havia um notch femoral após a ATJ.

Tabela 1 - Fatores de risco para fratura supracondiliana femoral pós-artroplastia total do joelho

\begin{tabular}{l}
\hline Osteoporose \\
\hline Doenças inflamatórias (AR, EA) \\
\hline Uso crônico de corticoides \\
\hline Doenças neurológicas \\
\hline Rigidez articular \\
\hline Manipulação sob anestesia \\
\hline Defeito cortical anterior (notch) \\
\hline Navegação \\
\hline Osteólise \\
\hline Cirurgia de revisão \\
\hline AR = artrite reumatoide, EA = espondilite ancilosante.
\end{tabular}

Recentemente foi publicado um artigo descrevendo uma FxP femoral no local de fixação do parafuso usado em cirurgia de navegação ${ }^{(24)}$ colocando mais um argumento na controvérsia sobre vantagens e desvantagens dessa técnica em ATJ.
Há diversas classificações das FxP de fêmur, desde Neer et $a l^{(25)}$, DiGioia e Rubash ${ }^{(26)}$, Chen et al ${ }^{(27)}$, Rorabeck e Taylor ${ }^{(28)}$ e finalmente Backstein et al ${ }^{(29)}$. A classificação de Rorabeck, como ficou conhecida, é a mais utilizada nas publicações recentes sobre o tema; divide as FxP em três categorias, de acordo com o componente femoral da ATJ - em tipo I, se estiver fixo; tipo II, se estiver solto; e tipo III, se houver perda óssea significativa do fêmur distal.

O objetivo do tratamento é naturalmente restituo ad integrum, o retorno às condições pré-fratura ou ainda melhores, nos casos em que já havia alguma complicação com a ATJ. Contudo, esse objetivo não é alcançado em grande número de vezes, e alguns autores estabeleceram metas mais modestas. Segundo Cain et $a^{(30)}$, um bom resultado seria ausência de dor, consolidação da fratura, alguma capacidade de marcha e arco de mobilidade do joelho a 90 graus de flexoextensão. Rorabeck et al $^{(31)}$ consideraram aceitável como resultado do tratamento de FxP femoral até dois centímetros de encurtamento, desvio do eixo varo-valgo de até cinco graus e de 10 graus no plano sagital.

\section{TRATAMENTO}

A maioria dos pacientes que sofre FxP é de idade avançada ou é portadora de doenças inflamatórias poliarticulares, e muitos apresentam comorbidades que fazem com que imobilização prolongada possa agravar seu estado geral de saúde e comprometer seu retorno a uma vida ativa, em nível semelhante a que tinham antes da fratura. Em nosso serviço, a idade média de ATJ primária nos pacientes portadores de artrose, a principal indica- 
ção deste procedimento, é de 71 anos. Por outro lado, FxP estão muitas vezes associadas à soltura asséptica ou osteólise, que são complicações de incidência a longo prazo. É fácil perceber que grande parte dos pacientes com FxP são octogenários, e precisam de reabilitação precoce após a fratura para evitar os problemas da perda de função de um membro inferior.

Em nosso serviço, devido ao número significativo de FxP atendidas, criamos modificação da classificação de Rorabeck e Taylor ${ }^{(28)}$ com objetivo de incluir novos tipos de fraturas que têm incidência progressivamente maior, e ainda definir os modos de tratamento mais apropriados para cada situação (Tabela 2).

Tabela 2 - Classificação HC- Curitiba das fraturas periprotéticas femorais após artroplastia total do joelho

\begin{tabular}{l|l}
\hline Tipo 1 & Prótese femoral fixa, bom estoque ósseo \\
\hline & a) Fratura estável \\
\hline & b) Fratura instável \\
\hline Tipo 2 & $\begin{array}{l}\text { Fratura + falha da prótese por soltura asséptica ou instabilidade, com } \\
\text { bom estoque ósseo }\end{array}$ \\
\hline Tipo 3 & Fratura + falha da prótese, estoque ósseo ruim \\
\hline Tipo 4 & Fratura no nível da extremidade da haste intramedular de revisão \\
\hline
\end{tabular}

Nas fraturas tipo 1a pode ser instituído tratamento conservador que, em geral, apresenta boa evolução. Meta-análise com 195 FxP supracondilianas femorais não desviadas e estáveis mostrou bons resultados em 83\% dos pacientes, comparando com $64 \%$ de sucesso como resultados de tratamento cirúrgico. Os objetivos do tratamento eram retorno ao estado pré-fratura, com joelho indolor, estável e com mobilidade articular funcional.

Fraturas tipo 1b representam a maior parte dos casos após a ATJ primária e são objeto de grande número de publicações, com técnicas criativas para solucionar esse problema de ocorrência cada vez mais frequente. O tratamento recomendado é a redução da fratura e osteossíntese estável. A razão da multiplicidade de artigos descrevendo diferentes técnicas cirúrgicas é a relativa fragilidade óssea da maioria dos pacientes que sofrem FxP, levando a grande número de insucessos no passado. Houve nítida evolução nos métodos de osteossíntese desde a época de fios de Steinmann cruzados, passando para placas condilares e implantes tipo DCS, com relatos de resultados irregulares e de complicações frequentes. Atualmente, as opções preferenciais são por métodos mais estáveis e menos invasivos, como hastes intramedulares bloqueadas, introduzidas por via retrógrada, ou placas tipo LISS, através de pequenas incisões com menor morbidade cirúrgica permitindo recuperação mais rápida ao paciente.
As hastes intramedulares apresentam duas limitações em relação às placas: em fraturas de traço bem distal podem ter maior limitação pela posição dos orifícios para os parafusos distais, e não podem ser utilizados nos casos em que a prótese femoral é do tipo PS (com estabilizador posterior) em que a caixa onde o poste tibial se aloja é fechada por metal. Os novos modelos de próteses femorais PS têm essa caixa aberta, prevendo esse problema técnico, mas as FxP ocorrem muitas vezes em pacientes que têm implantadas próteses há vários anos.

O grau de cominuição da fratura e a osteoporose acentuada em alguns pacientes fizeram com que alguns autores descrevessem técnicas para aumentar a estabilidade local, através de enxerto ósseo homólogo ${ }^{(32)}$ ou ainda cimento acrílico em combinação com haste ou placa ${ }^{(33)}$.

$\mathrm{O}$ tipo 2 de FxP femorais é o menos frequente. $\mathrm{Na}$ prática, quando a prótese está solta ou instável, existe perda óssea concomitante e, muitas vezes, o achado intraoperatório a converte para tipo 3. Quando ocorre, o tratamento adequado é a retirada da prótese, preservando-se o máximo de estoque ósseo possível e a revisão por novo componente com haste intramedular longa. Essa fixação pode ser completada por cabos metálicos ou fios de cerclagem, uma placa com parafusos e, eventualmente, enxerto ósseo.

As fraturas do tipo 3 necessitam de reposição do fêmur distal, e a decisão entre utilizar um aloenxerto ósseo maciço de fêmur distal ou uma prótese para tumores depende das características do paciente. Se este está em boas condições clínicas e perspectiva de sobrevida longa e ativa, é preferível o uso de enxerto de fêmur distal. O tempo de consolidação é longo e manterá o paciente com carga parcial leve no membro operado por seis meses. A vantagem é que se trata uma opção biológica, e havendo consolidação a perspectiva é de bons resultados em médio prazo $^{(34)}$. Além disso, há possibilidade de fixação das estruturas capsuloligamentares do joelho ao enxerto, permitindo o uso de próteses semiconstritas (Figura 3).

No caso do paciente ser de idade avançada, com comorbidades que demandam mobilização precoce com carga no membro operado, estado de demência ou outra situação que torne improvável que o paciente seguirá as recomendações médicas de apoio restrito, é recomendável o uso de próteses de fêmur distal do tipo empregado em cirurgia de tumores. Este tipo de implante tem um sistema de dobradiça que dispensa a função dos ligamentos do joelho, é fixado nos canais medulares do fêmur e da tíbia com hastes cimentadas e, portanto, permitem ao paciente fazer apoio total no membro inferior operado no dia seguinte à operação. Como desvantagens, são 

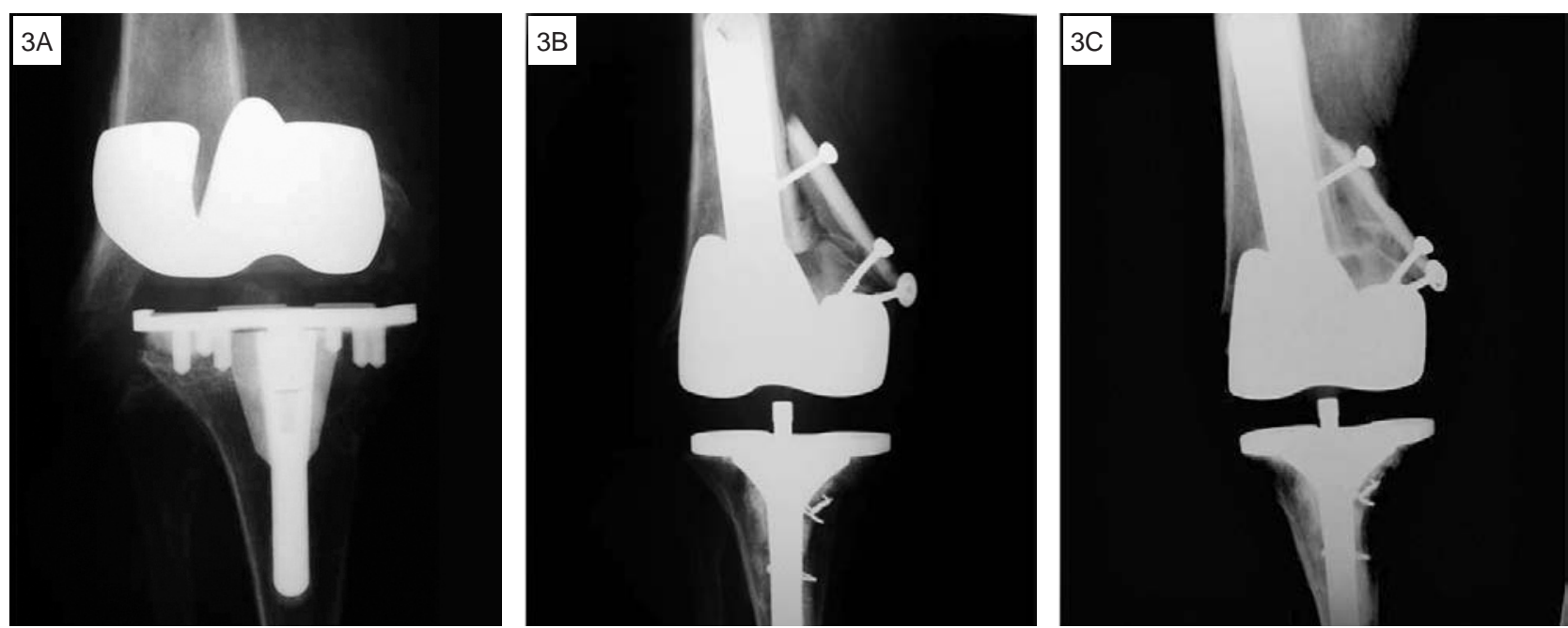

Figura 3 - A) Fratura periprotética de fêmur distal tipo 3. B) Revisão da ATJ e uso de enxerto homólogo maciço de fêmur distal, complementado com tábua óssea cortical. C) Aspecto com três anos de pós-operatório, mostrando consolidação do enxerto

próteses de histórico de resultados não satisfatórios a médio e longo prazos, com incidência elevada de complicações como soltura asséptica, quebra de material ou nova fratura na ponta da haste intramedular cimentada. Deve ser considerado ainda que, em caso de infecção profunda pós-operatória, há probabilidade de a solução final ser a amputação do membro inferior.

De fato a cirurgia de substituição do fêmur distal é uma operação rápida e pode ser aplicada em outros tipos de fratura em que o paciente não tem condições clínicas de suportar um período longo utilizando andadores ou muletas, podendo ser comparada ao caso de fraturas de colo femoral em que é utilizada uma prótese ao invés de osteossíntese em situações complexas, para evitar complicações clínicas em pacientes selecionados. Contudo, pela gravidade das complicações potenciais, seu uso deve ser bastante restrito (Figuras 4 a, b, c, d).

As fraturas tipo 4 representam um maior desafio téc- nico, pela presença da haste intramedular do componente femoral de revisão, limitando o uso de material de osteossíntese convencional. Estão descritos na literatura pertinente algumas possibilidades de resolução, como substituir a haste intramedular por outra mais longa ${ }^{(35)}$ ou o uso de placas ósseas corticais cercando o foco de fratura $^{(36)}$. O uso de placas parece ser o mais indicado nessas situações, seja do tipo LISS com parafusos unicorticais rosqueados na placa ou do tipo Dall-Miles, em que é usada uma combinação de cerclagens com cabo de aço na extremidade da fratura em que a haste intramedular está presente e parafusos no lado proximal. Nos casos de osteoporose, em que a fixação dos parafusos possa ficar comprometida, pode-se utilizar uma placa óssea na cortical medial, que serve como anteparo, melhorando a fixação dos parafusos e, adicionalmente, incorpora ao osso do paciente, reduzindo a possibilidade de refratura (Figuras 5 a, b, c).
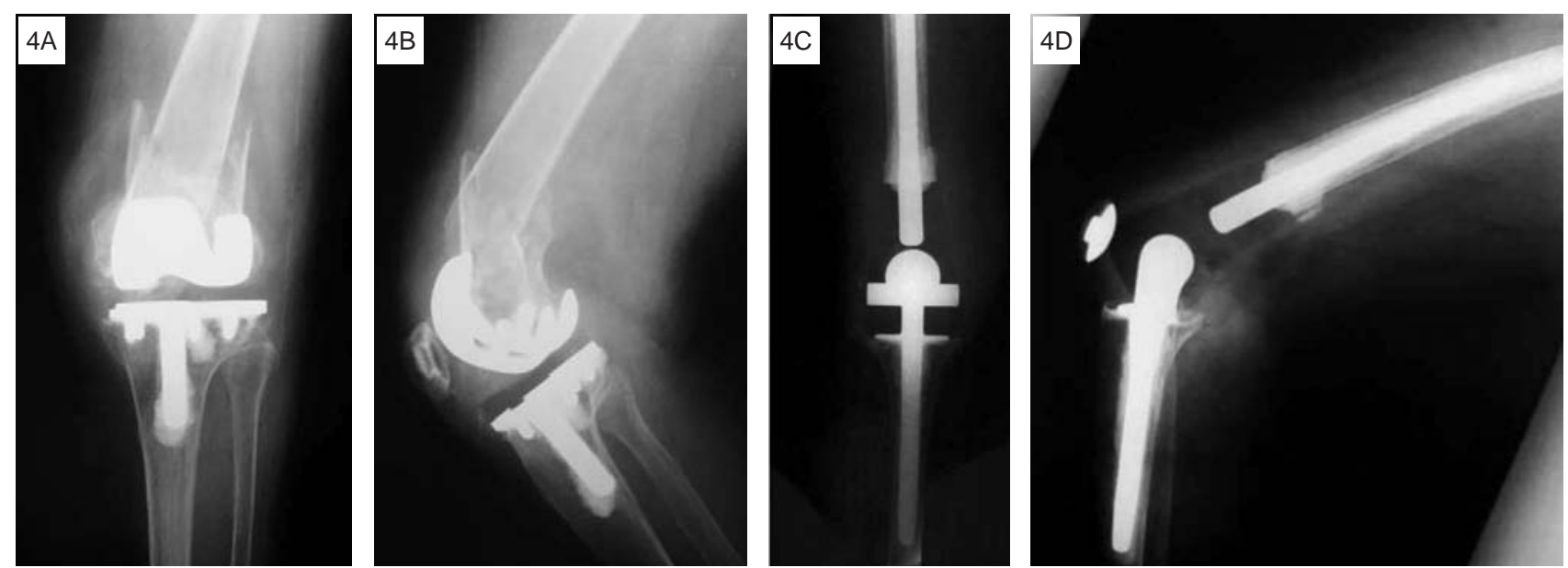

Figura 4 - A, B) Fratura periprotética de fêmur distal tipo 3 em paciente de 86 anos de idade, incidências de frente e perfil. C, D) Revisão com endoprótese não convencional 

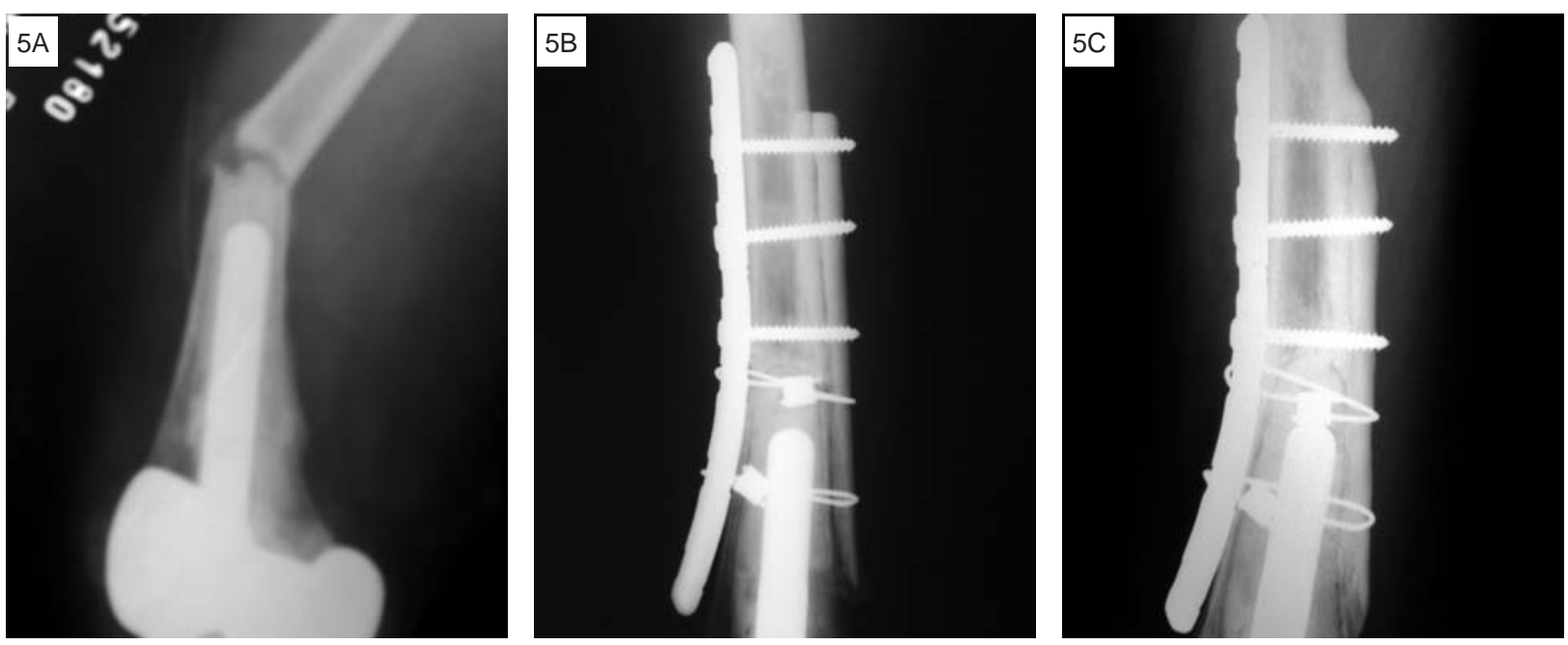

Figuras 5-A) Fratura periprotética tipo 4. B) Fixação com placa de Dall-Miles e tábua óssea medial, fixada com cabos e parafusos. C) Controle com cinco anos de pós-operatório

\section{CONCLUSÃO}

Pelo aumento do número de ATJ primária e perspectivas de maior longevidade dos pacientes, fraturas periprotéticas em joelho tornaram-se um problema de maior prevalência nos dias atuais. Uma abordagem sistemática de cada caso, disponibilidade de meios modernos de osteossíntese, de próteses com diversas opções de modelos e graus de constrição e acesso a banco de ossos, são recursos necessários para o tratamento adequado dessas fraturas complexas, visando manter a qualidade de vida e função dos nossos pacientes.

\section{REFERÊNCIAS}

1. Grace JN, Sim FH. Fracture of the patella after total knee arthroplasty. Clin Orthop Relat Res. 1988;(230):168-75.

2. Cameron HU, Fedorkow DM. The patella in total knee arthroplasty. Clin Orthop Relat Res. 1982;(165):197-9.

3. Brick GW, Scott RD. The patellofemoral component of total knee arthroplasty. Clin Orthop Relat Res. 1988;(231):163-78

4. Scott RD, Turoff N, Ewald FC. Stress fracture of the patella following duopatellar total knee arthroplasty with patellar resurfacing. Clin Orthop Relat Res. 1982;(170):147-51.

5. Rorabeck $\mathrm{CH}$, Angliss RD, Lewis PL. Fractures of the femur, tibia, and patella after total knee arthroplasty: decision making and principles of management. Instr Course Lect. 1998;47:449-58.

6. Bourne RB. Fractures of the patella after total knee replacement. Orthop Clin North Am. 1999;30(2):287-91.

7. Berman AT, Reid JS, Yanicko DR Jr, Sih GC, Zimmerman MR. Thermally induced bone necrosis in rabbits. Relation to implant failure in humans. Clin Orthop Relat Res. 1984;(186):284-92.

8. Willert HG, Ludwig J, Semlitsch M. Reaction of bone to methacrylate after hip arthroplasty: a long-term gross, light microscopic, and scanning electron microscopic study. J Bone Joint Surg Am. 1974;56(7):1368-82.

9. Figgie HE 3rd, Goldberg VM, Figgie MP, Inglis AE, Kelly M, Sobel M. The effect of alignment of the implant on fractures of the patella after condylar total knee arthroplasty. J Bone Joint Surg Am. 1989;71(7):1031-9.

10. Lynch AF, Rorabeck CH, Bourne RB. Extensor mechanism complications following total knee arthroplasty. J Arthroplasty. 1987;2(2):135-40.

11. Tharani R, Nakasone C, Vince KG. Periprosthetic fractures after total knee arthroplasty. J Arthroplasty. 2005;20(4 Suppl 2):27-32.

12. Tria AJ Jr, Harwood DA, Alicea JA, Cody RP. Patellar fractures in posterior stabilized knee arthroplasties. Clin Orthop Relat Res. 1994;(299):131-8.

13. Ortiguera CJ, Berry DJ. Patellar fracture after total knee arthroplasty. J Bone Joint Surg Am. 2002;84(4):532-40.

14. Insall JN, Lachiewicz PF, Burstein AH. The posterior stabilized condylar prosthesis: a modification of the total condylar design. Two to four-year clinical experience. J Bone Joint Surg Am. 1982;64(9):1317-23.

15. Engh GA, Ammeen DJ. Periprosthetic fractures adjacent to total knee implants: treatment and clinical results. Instr Course Lect. 1998;47:437-48.

16. Healy WL. Tibia fractures below total knee arthroplasty. In: Insall JN, Scott WN, Scuderi GR, editors. Current concepts in primary and revision total knee arthroplasty. Philadelphia: Lippincott; 1996. p.163-7.

17. Rand JA, Coventry MB. Stress fractures after total knee arthroplasty. J Bone Joint Surg Am. 1980;62(2):226-33.

18. Felix NA, Stuart MJ, Hanssen AD. Periprosthetic fractures of the tíbia associated with total knee arthroplasty. Clin Orthop Relat Res. 1997;(345):113-24.

19. Murase K, Crowninshield RD, Pedersen DR, Chang TS. An analysis of tibial component design in total knee arthroplasty. J Biomech. 1983;16(1):13-22.

20. Whiteside LA, Pafford J. Load transfer characteristics of a noncemented total knee arthroplasty. Clin Orthop Relat Res. 1989;(239):168-77.

21. Berry DJ. Epidemiology: hip and knee. Orthop Clin North Am. 1999;30(2):183-90.

22. Johansson JE, McBroom R, Barrington TW, Hunter GA. Fracture of the ipsilateral femur in patients wih total hip replacement. J Bone Joint Surg Am. 1981;63(9):1435-42.

23. Ritter MA, Faris PM, Keating EM, Meding JB. Postoperative alignment of total knee replacement. Its effect on survival. Clin Orthop Relat Res. 1994;(299):153-6.

24. Wysocki RW, Sheinkop MB, Virkus WW, Della Valle CJ. Femoral fracture through a previous pin site after computer-assisted total knee arthroplasty. J Arthroplasty. 2008;23(3):462-5.

25. Neer CS 2nd, Grantham SA, Shelton ML. Supracondylar fracture of the adult femur. A study of one hundred and ten cases. J Bone Joint Surg Am. 1967;49(4):591-613.

26. DiGioia AM 3rd, Rubash HE. Periprosthetic fractures of the femur after total knee arthroplasty. A literature review and treatment algorithm. Clin Orthop Relat Res. 1991;(271):135-42.

27. Chen F, Mont MA, Bachner RS. Management of ipsilateral supracondylar fêmur fractures following total knee arthroplasty. J Arthroplasty. 1994;9(5):521-6.

28. Rorabeck $\mathrm{CH}$, Taylor JW. Classification of periprosthetic fractures complicating total knee arthroplasty. Orthop Clin North Am. 1999;30(2):209-14.

29. Backstein D, Safir O, Gross A. Periprosthetic fractures of the knee. J Arthroplasty. 2007;22(4 Suppl 1):45-9.

30. Cain PR, Rubash HE, Wissinger HA, McClain EJ. Periprosthetic femoral fractures following total knee arthroplasty. Clin Orthop Relat Res. 1986;(208):205-14.

31. Rorabeck $\mathrm{CH}$, Angliss RD, Lewis PL. Fractures of the femur, tibia, and patella after total knee arthroplasty: decision making and principles of management. Instr Course Lect. 1998;47:449-58.

32. Alencar PGC. Proposta de tratamento com enxerto ósseo cortical homólogo para a fratura distal do fêmur pós-artroplastia total do joelho. Rev Bras Ortop. 2001;36(6):230-4

33. Bobak P, Polyzois I, Graham S, Gamie Z, Tsiridis E. Nailed Cementoplasty: A Salvage Technique for Rorabeck Type II Periprosthetic Fractures in Octogenarians. J Arthroplasty. 2009 Sep 22. [Epub ahead of print]

34. Backstein D, Safir O, Gross A. Management of bone loss: structural grafts in revision total knee arthroplasty. Clin Orthop Relat Res. 2006;446:104-12.

35. Mody BS, Khandelwal AO, Wala AC. Periprosthetic Femoral Fracture After a Well-Fixed Revision Total Knee Arthroplasty Treated With In Situ Effective Lengthening of Prosthesis. J Arthroplasty. 2009 Sep 1. [Epub ahead of print]

36. Tsiridis E, Spence G, Gamie Z, El Masry MA, Giannoudis PV. Grafting for periprosthetic femoral fractures: strut, impaction or femoral replacement. Injury. 2007;38(6):688-97. 